ArtefaCToS. Revista de estudios de la ciencia y la tecnología

eISSN: $1989-3612$

Vol. 7, No. 2 (2018), 2a Época, 9-33

DOI: http://dx.doi.org/10.14201/art201872933

\title{
Mitos y falsedades sobre el realismo científico
}

\section{Myths and Falsehoods about Scientific Realism}

\author{
Alberto CORDERO \\ City University of New York \\ Graduate Center \& Queens College \\ acordelec@outlook.com
}

Recibido: 13/05/2018. Revisado: 06/06/2018. Aceptado: 06/06/2018

\section{Resumen}

Los proyectos selectivistas tratan de fortalecer el realismo científico, pero influyentes sectores de pensamiento (tanto anti-realistas como realistas) consideran que sus logros son no solo problemáticos, sino desatinados, porque desfiguran y devalúan el realismo, transformándolo en una posición que no vale la pena tomar en cuenta. Un examen de los diversos cargos formulados a tal efecto y sus respectivos antecedentes históricos sugiere, sin embargo, que los cargos en cuestión descansan todos en mitos y/o falsedades sobre lo que caracteriza a una posición como realista y que, en tal medida, fracasan como objeciones.

Palabras clave: realismo científico selectivo; selectivismo; representación científica.

\begin{abstract}
Selective realist projects seek to strengthen scientific realism, but influential reactions (from anti-realist as well as realist camps) regard selectivist achievements as problematic and misguided developments, because - the story goesthose developments disfigure and devalue realism, transforming it into a position that is not worth taking into account. An examination of the charges formulated against selective realism and their respective historical background suggests, however, that the charges in question rest in myths and falsehoods about the character of realist positions; if so, the charges fail.
\end{abstract}

Keywords: selective scientific realism; selectivism; scientific representation. 


\section{Introducción}

Hay numerosas caracterizaciones del "realismo científico". En este artículo, el término subraya las siguientes tres tesis: (a) Existe una realidad independiente de la mente y sus representaciones; (b) el objetivo de las ciencias naturales es describir y explicar esa realidad; y (c) métodos forjados a lo largo de la práctica científica permiten realizar dicho objetivo en medidas que varían según cada campo. Se puede ser realista ontológico, epistemológico, semántico, metodológico, axiológico, pragmático, o múltiple. Muchos realistas incorporan todos los rubros, como por ejemplo Mario Bunge (2007).

En la práctica científica y la vida ordinaria, la actitud "natural” ante las explicaciones exitosas es de tipo realista. Por ejemplo, prácticamente todas las personas informadas aceptan la teoría según la cual Encélado, el satélite de Saturno, tiene un océano de agua subterráneo. Asienten a esta propuesta, inicialmente tan extravagante, porque las inferencias y corroboraciones experimentales que llevan a ella convencen.

Típicamente, una teoría versa sobre cierto dominio empírico, cuyo comportamiento explica en términos de entidades y procesos postulados para tal fin. Los científicos tienden a considerar verdaderas (al menos en alguna medida) las teorías que conducen sistemáticamente a predicciones novedosas inesperadas. Los dictámenes involucrados son complejos, sin embargo. Si nos guiamos por la historia de la ciencia, el éxito empírico de una teoría no indica necesariamente que las entidades y procesos postulados por ella existen o son aproximadamente como dice. Por citar un caso, la concepción de la luz de Fresnel, basada en la idea de un peculiar "éter luminífero", impresionó en su momento a todos por sus notables explicaciones y predicciones, tanto que la existencia del éter llegó considerarse cosa segura en el siglo XIX. Pero luego, en el siglo XX, los físicos concluyeron que el tal éter no existe. Casos análogos abundan en la historia (Vickers, 2013). No comprometen fatalmente la opción realista pero sí la obligan a sobriedad. En particular, las evidencias sugieren que, si bien las explicaciones empíricamente exitosas develan verdades, no develan toda la verdad ni nada más que la verdad (realismo ingenuo). Por ello, todos admitimos que la relación entre el éxito empírico de una teoría y su contenido de verdad es compleja. Matizando las tesis tradicionales, ahora los realistas científicos tratan de ligar el éxito de una teoría a la veracidad de solo ciertas partes de ella, no a toda su trama descriptiva. Es tarea de los realistas identificar convincentemente dichas partes y sus respectivas credenciales de veracidad.

La mencionada "actitud natural" sobre el realismo habitualmente ignora estas sutilezas. En parte por dicha razón, algunos sectores de la cultura contemporánea rechazan las interpretaciones realistas. Las críticas que hacen incurren a menudo en generalizaciones escépticas, conspicuamente en el caso de pensadores que ven los proyectos realistas como "meta-narrativas" ingenuas, indignas de personas 
adultas $^{1}$. Análogamente, para no pocos sociólogos y políticos de la ciencia, el éxito empírico, la objetividad y la veracidad de las explicaciones deben entenderse ante todo como constructos sociales (y los argumentos racionales, como combates políticos $)^{2}$. Tales radicalismos no agotan las fuentes de suspicacia contra la ciencia en las esferas académicas. Mucho del actual fuego negativista viene de desarrollos dentro de la filosofía analítica en el siglo XX. Irónicamente, estas y otras displicencias anti-realista ocurren justo cuando las ciencias "duras" muestran logros intelectuales y prácticos sin precedentes en la historia, incluyendo una cartografía de la realidad que hoy todos aprovechamos con voracidad.

Popular o no, el proyecto realista sigue en pie. Desde los años ochenta, realistas de diverso cuño (notablemente, Mary Hesse (1974), Dudley Shapere (1983), Ronald Giere (2006), Philip Kitcher (1993), Jarrett Leplin (1997), y Stathis Psillos (1999), y Ernan McMullin (2013), entre muchos otros, aceptan que la observación científica trae carga teórica y que las descripciones científicas y el lenguaje en general están mediatizados por intereses humanos. Tomando el toro por las astas, estos realistas buscan mostrar la compatibilidad de las dependencias notadas con versiones fuertes de los ideales de objetividad y verdad científicas. Los proyectos que proponen reconocen la existencia de marcos epistémicos socialmente construidos, pero también la idea de que dichos marcos, lejos de impedir nuestro acceso epistémico al mundo externo y a la verdad pública, los posibilitan. Muchas de las críticas a estas propuestas realistas se centran en problemas técnicos - e.g., en torno a las ideas de éxito teórico, compromiso realista, y verosimilitud.

Los debates correspondientes continúan en marcha. Mi tema en este artículo se concentra en un género de objeciones relacionado pero distinto, aplicado sobre todo a proyectos que dejan de lado el énfasis tradicional en teorías completas en favor de un realismo enfocado hacia fragmentos teóricos limitados (realismo selectivo). Se trata de objeciones corrosivas, avaladas por influyentes críticos (incluyendo algunos realistas), para quienes los proyectos selectivistas hacen demasiadas concesiones al anti-realismo y, en cualquier caso, carecen de relevancia, pues - sostienen - aun si lograsen superar sus dificultades técnicas a lo mucho pueden dar lugar a posiciones débiles que "no vale la pena tomar en serio". En lo que sigue, sugeriré que lo que no vale la pena tomar en serio son las objeciones del género mencionado, porque descansan en mitos (alegorías de verdades sig-

\footnotetext{
${ }^{1}$ Jean-François Lyotard (1979) define la postura posmoderna como incredulidad hacia las "meta-narrativas", que en su opinión incluyen la Ilustración, la objetividad externa y el realismo científico.

${ }^{2}$ Este tipo de sociologismo es aparente, por ejemplo, en la página web del Centro de Harvard para Estudios de la Ciencia y la Tecnología (STS) < http://sts.hks.harvard.edu/about/whatissts. html >. Para una crítica panorámica de las ofertas sociologistas y postmodernistas radicales sobre la ciencia ver Alan Sokal and Jean Bricmont (1998).
} 
nificativas que tomados literalmente resultan fatales) y/o falsedades. Pero antes, hace falta considerar el trasfondo del actual debate sobre el realismo científico. Es el tema del resto de esta primera parte.

La estructura del presente artículo es como sigue. La sección siguiente esboza el ideal del realismo científico inmerso en proyectos clásicos como el de William Whewell. La sección 1.2 comenta el impacto que sobre estos proyectos tuvieron los grandes cambios conceptuales de la física en las primeras décadas del siglo XX y las secuelas representadas, primero por el positivismo lógico y -tras la caída de éste- el surgimiento de ideas historicistas neo-románticas como las de Thomas Kuhn, seguidas de reacciones objetivistas como las del Empirismo Constructivo y la lectura escéptica de la historia de la ciencia. Las secciones 1.3 y 1.4 presentan la estrategia general del realismo selectivo como un ejemplo de respuesta contemporánea, así como su recepción crítica. La sección 1.5 considera ciertos supuestos populares acerca del realismo científico. La segunda parte del artículo analiza y rechaza como falsos y desorientados los supuestos delineados en la sección anterior $-\mathrm{y}$, con ellos, la acusación de que el selectivismo diluye el proyecto realista convirtiéndolo en algo que no vale la pena defender.

\subsection{Un poco de historia}

A mediados del siglo XIX, la actitud realista frente a las teorías exitosas parecía inquebrantable. Concepciones como la del teólogo, minerólogo, historiador y filósofo de la ciencia William Whewell atestiguan el optimismo de la época. La ciencia inductiva, argumentaba Whewell, progresa epistémicamente porque las teorías se toman en serio solo si pasan pruebas rigurosas. Una teoría convence - sostenía- en virtud de su poder predictivo, su coherencia, y la "consiliencia de inducciones" que logra aparejar. A Whewell le parecía particularmente importante la predicción de hechos desconocidos, porque - enfatizaba- confiere una credibilidad mayor que la explicación de hechos ya conocidos (1858, Aforismo XII). Llamaba "consiliencia" a la convergencia que ocurre cuando una inducción, obtenida a partir de una serie de hechos, coincide con otra inducción basada en otra serie diferente de hechos (por ejemplo, las inducciones newtonianas que, desde diversos ángulos, apuntan a la existencia de una fuerza atractiva inversamente proporcional al cuadrado de la distancia de separación de los cuerpos materiales como causa de diferentes clases de fenómenos). En cuanto a la "coherencia", según Whewell la hay cuando, sin introducir modificaciones ad hoc, es posible extender una hipótesis a una clase de fenómenos no tenidos cuenta al armar la hipótesis, como cuando Newton extiende su teoría de la gravitación a la "actividad de las mareas". Una hipótesis con logros en los tres rubros convence de su veracidad, argüía Whewell:

En toda la historia de la ciencia, en la medida en que yo sepa, no se puede señalar ningún ejemplo en que la Consiliencia de Inducciones 
haya dado testimonio en favor de una hipótesis descubierta posteriormente como falsa. [...] Cuando la hipótesis, por sí misma y sin ajustes, nos da la regla y la razón de una clase de hechos no contemplados en su construcción, tenemos un criterio de realidad que nunca ha favorecido la Falsedad. (1847, 67-68)

Los científicos del siglo XIX estaban mayoritariamente de acuerdo--en particular Charles Darwin, tenaz en sus esfuerzos para que la teoría de la selección natural satisficiera al menos las condiciones de coherencia y consiliencia ${ }^{3}$. Al entrar el siglo en su último cuarto, la mecánica newtoniana (incluyendo la Ley de la Gravitación), el electromagnetismo clásico y la química de Lavoisier, calificaban holgadamente en términos de los criterios de Whewell — nada, se pensaba, podría poner en duda las concepciones básicas de dichas teorías. Sin embargo, esta confianza en el poder epistemológico de la ciencia, así como el optimismo de Whewell sobre el progreso, estaban a punto de colapsar bajo las profundas revisiones conceptuales que ocurrirían muy pronto en la física.

En las primeras décadas del siglo XX, el descubrimiento de fenómenos difíciles de explicar mediante la física clásica puso al descubierto limitaciones en las teorías recibidas. Súbitamente, la mecánica newtoniana parecía descansar en supuestos erróneos sobre la naturaleza de la masa, la gravitación, la materia, la causalidad, la separabilidad, la identidad, y más. La confianza en la capacidad del pensamiento científico para inferir teorías veraces a partir del éxito empírico se desplomó. La imaginación científica empezó a verse como una facultad demasiado fértil y poco fiel a las profundidades del mundo. Interpretar realistamente las teorías fundamentales perdió plausibilidad. Reacciones epistemológicas radicales, que recomendaban afanosas purgas empiristas, cobraron fuerza, llevando eventualmente a la constitución del llamado "empirismo lógico" como filosofía dominante de la ciencia. Esta posición se caracterizó por privilegiar la información de nivel "bajo" (ordinario o basada en datos de los sentidos) y desconfiar de la inferencia abductiva y la especulación teórica en general. Para los empiristas lógicos, el éxito científico indicaba progreso instrumental pero no avance del conocimiento teórico. El empirismo lógico se derrumbaría pocas décadas después bajo sus propias dificultades lógicas y falsedades históricas. Cuando vino la caída, sin embargo, las reacciones no apuntaron mayoritariamente hacia el realismo, sino más bien lo contrario.

\subsection{Proyectos neo-románticos y neo-positivistas}

Las críticas al empirismo lógico trajeron a primer plano el carácter problemático de la observación con respecto a la verdad y su dependencia irreducible de

\footnotetext{
${ }^{3}$ Ver e.g. Ruse (1979, Capítulo 7). Los argumentos darwinianos no lograron satisfacer el requisito de predicción hasta finales del siglo siguiente (Cordero 2011a).
} 
presupuestos teóricos. Ambos aspectos llevaron a muchos pensadores a extraer consecuencias a primera vista calcinantes para el ideal tradicional de objetividad. De modo particularmente influyente, en su obra La Estructura de las Revoluciones Cientificas (1962), Thomas S. Kuhn extendió a la observación científica la desconfianza que los empiristas habían reservado para las teorías. En dicho libro, Kuhn presenta la observación como algo tan fuertemente dependiente de supuestos teóricos como para permitirse afirmar que los científicos adscritos a teorías diferentes viven "en mundos diferentes" (1962, 116-8). Según Kuhn, era imposible comparar objetivamente los logros de teorías propiamente distintas - no hay, decía, perspectiva neutra que permita hacer tal comparación; en su opinión, las teorías diferentes debían considerarse "inconmensurables”. El "anarquismo epistemológico" de Paul Feyerabend en Contra el Método complementaba esta veta de opinión neo-romántica con apuestas a la introducción de recursos irracionales en la investigación científica.

Una componente especialmente contraria al realismo científico en las obras de Kuhn y Feyerabend es la visión holista del lenguaje y la trama conceptual que él defendía, según la cual, por ejemplo, si la mecánica de Einstein es verdadera, entonces la masa newtoniana (que es independiente de la velocidad) no existe y, por lo tanto, no representa nada. Desde su perspectiva, las descripciones científicas remiten a meras ficciones y, en consecuencia, el "mundo real" no puede (ni debe) ser una preocupación de la ciencia. Con el tiempo, Kuhn se distanció de esta versión extrema de su concepción, llegando a admitir que la selección de teorías científicas sigue criterios inter-teóricos informales, centrados en la satisfacción de valores compartidos como la exactitud, la coherencia, el alcance, la simplicidad y la fertilidad ${ }^{4}$. Sin embargo, nunca aceptó el proyecto del realismo científico, que consideraba comprometido con la tesis de convergencia teórica hacia la verdad absoluta. En la segunda edición de La Estructura, descarta explícitamente las esperanzas realistas por considerarlas incoherentes (1970, 206).

En los años setenta, prácticamente todos los estudiosos de la ciencia aceptaban que la observación y el método científico dependen de las teorías de base, las perspectivas y las circunstancias cognitivas de cada contexto histórico; muchos aceptaban también que la verdad no se da de manera simple y absoluta a ningún nivel. Sin embargo, en la filosofía de la ciencia (a diferencia de los estudios sociales), eran comparativamente pocos los que consentían las propuestas radicales de Kuhn y Feyerabend. Para la mayoría, el análisis filosófico, la historia y la ciencia mostraban que la observación tiene una dependencia teórica, pero también que la experiencia y la observación están subdeterminadas por las teorías -como lo sugiere la frecuencia con que la experiencia choca destructivamente con las teorías. La crítica del extremismo neo-romántico engendró reacciones objetivistas por parte de pensadores tanto anti-realistas como realistas.

${ }^{4}$ Ver e.g. "Objectivity, Value Judgment, and Theory Choice”, (Capítulo 13 en Kuhn, 1977). 
Las respuestas anti-realistas encontraron sus expresiones más influyentes en proyectos complementarios encabezados por Bas van Fraassen y Larry Laudan, respectivamente. Según van Fraassen (1980), las teorías científicas intentan dar un recuento literalmente verdadero de cómo es el mundo, pero - añade-aceptar una teoría no implica creer que ella es verdadera. En su opinión, lo filosóficamente pertinente es la adecuación empírica de las teorías a los fenómenos de interés, no la verdad teórica. De otro lado, según Laudan (1981; 1984), la historia de las teorías científicas deja al descubierto un enorme cementerio de propuestas que, en su momento, se consideraron exitosas pero que luego mostraron estar fundamentalmente equivocados. Esta apreciación lleva a Laudan a sostener que las mejores teorías actuales están también equivocadas (Inducción Pesimista).

El Empirismo Constructivo de Van Fraassen mantiene una postura agnóstica radical hacia lo que las teorías dicen sobre lo "no-observable", una restricción que resulta extrańa y artificial a muchos estudiosos de la ciencia ¿Dejan las teorías exitosas de ser epistémicamente confiables cuando hablan sobre aspectos del mundo inaccesibles a la percepción humana natural? ¿Por qué? Dadas las diferencias discernibles en los grados de confirmación de las diversas aserciones científicas, es razonable tratar de discriminar epistémicamente entre los contenidos de la ciencia. ¿Pero hacerlo usando como demarcación la frontera de lo perceptible? Las buenas razones para discriminar contenidos son específicas a cada situación. Por ejemplo, allí donde una teoría exhibe contenidos indiferentes a las pruebas experimentales disponibles (subdeterminación empírica "efectiva") resulta arbitrario tomar una posición realista en favor de dichos contenidos. Una situación así ocurre, por ejemplo, con la teoría de muchos mundos y la teoría GRW en la física cuántica (Cordero, 2001), donde dichas teorías describen mundos muy diferentes entre sí pero cuyas diferencias no son accesibles mediante la tecnología disponible. De lo que se trata, por consiguiente, es de identificar con cuidado las partes creíbles de una teoría exitosa. Pero esto no se puede hacer bajo lineamientos "globales" como los propuestos por van Fraassen, porque no hay acuerdo sobre los demarcadores epistemológicos a utilizar.

Por su parte, Laudan también responde a Kuhn afirmando la existencia de progreso científico, dentro de una lectura anti-realista de la ciencia y el conocimiento. La confiabilidad alcanzada por las teorías, sostiene Laudan, no indica que éstas develan lo que hay realmente en el mundo. La historia de la ciencia, nota, está repleta de explicaciones que, habiendo obtenido amplia comprobación empírica, fueron luego descartadas en favor de explicaciones dramáticamente diferentes sobre los constituyentes de la realidad. Incluso teorías con fuerte poder predictivo han tenido ese fatal destino, subraya. Los ejemplos de Laudan incluyen las teorías del calórico, el flogisto, y la ya mencionada óptica del éter luminífero, todas muy exitosas, cuyos términos centrales al final resultaron no referir a nada en el mundo físico-como en opinión de Laudan deberían hacerlo las teorías veraces. Éste y otros argumentos complementarios, que sistemáticamente apelan a la inestabilidad confirmacional de las descripciones teóricas "profun- 
das", forman la base de la "confutación" que Laudan ofrece del supuesto vínculo realista entre el éxito empírico y la verdad. Según Laudan, el realismo científico está tanto subdeterminado por los datos disponibles como refutado por la historia de la ciencia. En su opinión, la ciencia ofrece una creciente confiabilidad instrumental y metodológica, pero ni contribuye verdades teóricas ni las necesita para realizar sus hazańas. A la luz de la historia, advierte, no tenemos razón para confiar en lo que las teorías contemporáneas dicen sobre la "realidad profunda".

¿Por qué el mejoramiento de la confiabilidad instrumental no puede relacionarse con al menos algunas ganancias con respecto a la verdad teórica? Laudan no se pronuncia al respecto. Su anti-realismo descansa en ideas peculiares sobre teorías falsas, el lenguaje y cierta noción de "verdad aproximada" que toma de Karl Popper, ideas que los realistas consideran infundadas, como se discute en la siguiente sección.

\subsection{La resistencia realista}

Los proyectos anti-realistas esbozados en la sección anterior no son todos los existentes, pero sí quizá los más influyentes en el debate actual sobre el realismo científico. En el campo contrario, las respuestas dominantes incluyen los enfoques de "realismo selectivo" encabezados en los ańos noventa por Philip Kitcher, Jarrett Leplin, y Stathis Psillos.

Leplin, colaborador de Laudan en algunos proyectos objetivistas, ha mantenido una distancia crítica frente el anti-realismo de éste. La historia, señala, no dificulta el realismo en mayor medida que nuestra experiencia de objetos ordinarios es inequívocamente verídica (Leplin, 1984). A su juicio, solo las posiciones realistas explican la capacidad de las teorías para dilucidar y predecir exitosamente fenómenos que no fueron diseńadas para cubrir. Leplin admite que la continuidad conceptual a través de las revoluciones es un tema contencioso, pero enfatiza inducciones favorables al realismo como la siguiente:

[...] un patrón histórico que se ha mantenido estable a lo largo del cambio científico es la tenacidad de los juicios preferenciales sobre las teorías. Aunque una teoría que reemplaza a otra es luego reemplazada, su superioridad sobre la teoría precedente continúa siendo reconocida. Así como como la historia registra juicios sostenidos de la inaceptabilidad última de las teorías, en similar medida registra juicios estables sobre sus méritos relativos. [...] Tales juicios no se limitan a las dimensiones pragmáticas del éxito predictivo, sino que incluyen comparaciones explicativas. Newton dio una mejor explicación de la caída libre que Galileo, aunque ambas explicaciones han sido superadas. Si mantenemos tales juicios más allá de la tenencia de las teorías mismas, debemos considerar que una teoría ha obtenido más de los hechos pertinentes o que 
ha descrito esos hechos con mayor precisión, incluso si ambas teorías son falsas. Si las explicaciones propuestas por ambas teorías fueran rechazadas como totalmente desprovistas de verdad, tales comparaciones resultarían imposibles. $(1984,214)$

Según Leplin, hay tan buenas razones inductivas para concluir que las teorías científicas aumentan en contenido de verdad como para concluir que todas las teorías son falsas (en el sentido de no ser completamente verdaderas). En cualquier caso, agrega, la fidelidad descriptiva total rara vez es un objetivo en la ciencia real. Los propios científicos apelan rutinariamente a descripciones extraídas de teorías descartadas y que continúan aceptando como "aproximadamente verdaderas". Para los realistas, las ideas de Laudan distorsionan la forma en que los científicos comprenden el lenguaje y la verdad.

Una contestación anti-realista posible es que, incluso si se aceptara que las anteriores réplicas responden a Laudan, van Fraassen podría reformular la inducción de Leplin anti-realistamente (esto es, en términos de la mera adecuación empirica de las teorías). El resultado sería una afirmación más modesta (y por lo tanto más fácilmente creíble) sobre la tenacidad de juicios sobre los méritos relativos de teorías sucesivas. A su vez, los realistas podrían responder acusando de arbitrario y oscurantista el objetivo de adecuación empírica.

Por su lado, los kuhnianos niegan coherencia a todas estas escaramuzas realistas. Hoy, sin embargo, la mayoría los filósofos de la ciencia consideran sospechoso o incluso incoherente los holismos y relativismos propugnados por Kuhn, Feyerabend, y para tal caso W.V.O. Quine. Como sugiere Alexander Rosenberg (2000), los anti-realistas necesitan explicar, por ejemplo, el aparente acuerdo sobre las clases de cosas y regularidades que, de modo característico, "encuentran" en el mundo los pueblos pre-científicos. También necesitan explicar la velocidad con la cual dichos pueblos son capaces de desplazar su lealtad hacia las clases y las regularidades propuestas por la ciencia moderna. De otro lado, tampoco es cierto que los "términos teóricos" padezcan de inconmensurabilidad. Según han notado diversos críticos (e.g., Shapere 1984, Devitt 1991), prácticamente todos los términos teóricos entran al discurso científico ya dotados de cierto significado y conocimiento estable de base - la práctica científica asume conocimientos pre-teóricos, los cuales clarifica y mejora. En los grandes cambios teóricos la información y la comprensión previas no se pierden completamente al estilo kuhniano, sino que se revisan y generalizan críticamente.

Los realistas cuestionan, pues, las presunciones de Kuhn, Feyerabend y Laudan sobre el lenguaje y la verdad, arguyendo que el holismo lingüístico hace inexplicables las prácticas cognitivas y lingüísticas humanas, carece de evidencia positiva a su favor, y tiene como única base prejuicios meta-científicos sobre el lenguaje y el significado. Estos rechazos anuncian algunas estrategias realistas, 
particularmente en relación con la fragmentación de las teorías en partes epistemológicamente independizables y la identificación de partes veraces en teorías descartadas.

Un legado importante de esta fase del debate sobre el realismo científico es la centralidad que ahora tiene la cuestión sobre los límites de la inferencia ampliativa en las ciencias empíricamente exitosas. Todos los contendientes objetivistas mantienen posiciones realistas con respecto a las descripciones científicamente justificadas sobre aspectos del mundo accesibles a la percepción natural. La disputa en curso es sobre la razonabilidad de posiciones que extienden las inferencias justificadas a contenidos teóricos de la ciencia.

\subsection{El realismo selectivo y sus complicaciones}

De la década de los ochenta a la fecha, diferentes proyectos de realismo se han concentrado en la retención de "contenidos veraces" a través del cambio teórico. Un ejemplo de retención es el siguiente, mantenido en las teorías ópticas desde las primeras décadas del siglo XIX: La luz está hecha de ondulaciones transversales microscópicas que, en primera aproximación, satisfacen las leyes de Fresnel de la reflexión y la refracción ("Núcleo de Fresnel"). Los realistas presentan este caso como típico. Para ellos, aceptar que teorías falsas contienen partes veraces no crea automáticamente ningún problema conceptual. Propuestas de este tipo incluyen investigaciones perspectivistas (e.g. Giere, 2006) y los proyectos ya mencionados de realismo "selectivo".

Los selectivismos se dividen en proyectos situados entre dos extremos. Uno, liderado por John Worrall (1989) se concentra en estructuras matemáticas retenidas a lo largo de sucesiones teóricas (realismo estructural). El otro (realismo selectivo), representado en los años 90 por ciertos trabajos seminales (e.g., Kitcher 1993, Leplin 1997, y Psillos 1999), se concentra en contenidos teóricos más amplios, preservados a través del cambio teórico (entidades, procesos, historias naturales). Según los selectivistas, las teorías que, tras mostrar poder predictivo, son luego superadas, contienen no obstante partes veraces, al menos a nivel de entidades y estructuras de tipo "funcional" o "efectivo". Cuando una teoría pierde protagonismo, partes significativas de ella se mantienen vigentes en las teorías sucesoras, donde continúan mostrando éxito empírico y libertad de dudas específicas. Ese, aseguran los selectivistas, es el caso no solo del Núcleo de Fresnel sino de miríadas de partes teóricas heredadas de propuestas científicas a lo largo de los siglos. Por ejemplo, las partículas de la teoría cinética de la materia no existen, estrictamente hablando. Sí lo hacen en cambio, versiones funcionales de las mismas, entendidas como sistemas localizados de interacción, dotados de propiedades cuyos promedios cuánticos y máximos de probabilidad corresponden, en muchos casos, a relaciones clásicas. Lo mismo vale para numerosas historias evolutivas conjeturadas por Darwin y su círculo, legiones de estructuras y proce- 
sos microbiológicos, partes de la genética mendeliana, multitudes de estructuras moleculares, la gran mayoría de las entidades subatómicas consideradas "bien establecidas" en la segunda mitad del XX, así como innumerables redes causales, historias y entidades funcionales postuladas por teorías consideradas exitosas en términos de las predicciones novedosas a que dan lugar.

Los selectivistas no adoptan posiciones realistas acerca de teorías totales sino de partes específicas de las mismas. Cambian así el enfoque realista tradicional, concentrándolo en componentes teóricas identificadas mediante criterios de selección que presentan como "confiables". Desde esta perspectiva, el éxito empírico funciona como un marcador de veracidad bajo la tesis de que las teorías con claro poder predictivo describen correctamente al menos algunos aspectos del dominio de interés, incluyendo aspectos inaccesibles a la percepción pre-científica.

Los proyectos iniciales de Kitcher, Leplin y Psillos tuvieron buena acogida, pero mostraron fallas e incoherencias en las diversas reglas de selección propuestas. Éstas identificaban como contenidos veraces proyecciones retrospectivas de teorías presentes, o invocaban distinciones problemáticas entre partes "activas" (working) e "inactivas" (idle) dentro de las teorías. Una de las principales estrategias de Psillos se apoyaba en una noción cuestionable de "partes indispensables" 5 . El desarrollo del realismo selectivo no ha sido tarea fácil. De todos modos, hay en marcha iniciativas argüiblemente prometedoras - una "segunda ola" de selectivismos, que incorpora reformas, entre las que figuran propuestas como las de Juha Saatsi (2005), Ioannis Votsis (2011), Peter Vickers (2013), y Alberto Cordero (2017), entre otras. Cordero (2017), en particular, focaliza la selección de contenidos epistémicamente promisorios en partes teóricas que (a) están claramente implicadas en predicciones nuevas e inesperadas, (b) no presentan dudas específicas convincentes contra ellas, (c) reciben esclarecimiento confirmatorio a partir de teorías externas independientemente exitosas, y (d) muestran resistencia al cambio teórico. En esta propuesta, la convergencia de dichos logros se toma como un marcador del contenido de verdad en la teoría bajo escrutinio.

El proyecto selectivista no atrae intelectualmente a todos; algunos estudiosos de la ciencia se mantienen altamente críticos. Las discusiones pertinentes se encuentran en pleno desarrollo; pero de ese importante tema trato en otras publicaciones (e.g. Cordero, 2017). Este artículo se centra en un aspecto menos explícito (pero profundo) del debate actual sobre al realismo. Según una opinión - compartida por no pocos simpatizantes del realismo- el selectivismo es un proyecto contraproducente porque se articula a costa de debilitar la posición realista hasta convertirla en algo que "no vale la pena intentar". Quienes así piensan consideran que, incluso si el realismo selectivo lograse ofrecer un criterio de selec-

\footnotetext{
${ }^{5}$ Ver e.g. Lyons (2002).
} 
ción convincente, igual no interesaría, porque su propuesta contraviene aspectos que dan sentido, valor y vigencia al realismo como proyecto intelectual. Veamos los argumentos en detalle.

\subsection{Supuestos populares sobre el realismo}

Una queja contra las estrategias selectivistas es que violentan aspectos presuntamente claves del realismo científico, particularmente los siguientes:

(1) La unidad inquebrantable de las explicaciones ofrecidas por las teorías.

(2) La relación entre el éxito teórico y el logro de descripciones exactas y literalmente verdaderas.

(3) El ideal de las teorías como propuestas verdaderas en su mayor parte.

(4) La significación y enjundia realista de los términos "centrales" de una teoría.

(5) La universalidad de las leyes fundamentales.

(6) La ligadura tradicional entre el realismo y el progreso cognoscitivo.

(7) La inteligibilidad que la interpretación realista de una teoría aporta al dominio estudiado.

(8) La idea de que satisfacer los anteriores rasgos es condición necesaria para tomar en serio una propuesta realista.

Los proyectos selectivistas en curso dejan de lado la anterior lista de expectativas. En particular:

- Todos desplazan la atención realista hacia fragmentos descriptivos específicos considerados epistémicamente promisorios (en vez de concentrarse en el todo integral previsto por los autores de la teoría bajo consideración). El selectivista toma dichos fragmentos como "aproximadamente verdaderos" en la medida que, a la luz de razones específicas, concluye que contienen descripciones teóricas veraces -independientemente de si dichas descripciones son completas, parciales, diluidas, abstractas o de rango limitado.

- Los selectivistas aceptan que el mundo es solo rara vez (si acaso) "fiel y exactamente" como dicen las teorías exitosas. 
- Una teoría dotada de poder predictivo novedoso generalmente aumenta el saber teórico del mundo; pero no hay razón para esperar que sea "correcta en su mayor parte": lo que importa es si incrementa o no el conocimiento teórico.

- En términos selectivistas, una hipótesis exitosa contiene verdades teóricas novedosas, pero no necesaria ni mayormente al nivel descriptivo más fundamental o profundo. Los términos centrales de una teoría unifican los contenidos de ésta, pero la fuerza epistémica de dichos términos puede ser comparativamente débil.

- $\quad$ En el selectivismo, atribuir veracidad a enunciados teóricos generales no significa presumir que las leyes implicadas en ellos tienen aplicabilidad universal (irrestricta).

- Los selectivistas no esperan que las teorías empíricamente exitosas desplieguen progreso uniformemente convergente hacia una "descripción final”.

- En términos selectivistas, la inteligibilidad que una teoría confiere a su dominio es generalmente incompleta.

\section{Los supuestos bajo la lupa}

Los rasgos enunciados en la sección anterior ameritan un análisis detallado.

\subsection{Las teorías como constructos inquebrantables}

¿Es razonable pensar en las teorías como sistemas holistas y que tomar una posición realista con respecto a cualquiera de ellas conlleva aceptar como verdadero todo lo que dice? Al menos dos motivaciones animan estas ideas. Una es un híbrido entre la concepción enunciativa de las teorías y el holismo lingüístico ${ }^{6}$, constructo meta-científico favorecido en los años sesenta y setenta del último siglo por pensadores tan superficialmente diferentes como Ernest $\mathrm{Nagel}^{7}$ y Thomas Kuhn, mantenido en décadas subsiguientes por numerosos filósofos. Por ejemplo, la inducción pesimista de Laudan (1981) asume que las teorías científicas

\footnotetext{
${ }^{6}$ Según el enunciativismo, una teoría es un conjunto de enunciados, lógicamente estructurados, acerca de cierto dominio, cuyo comportamiento la teoría tiene por objetivo explicar y predecir. Según el holismo, el significado de una expresión depende radicalmente de la totalidad del sistema conceptual utilizado, de modo que no es posible entender propiamente una expresión sin considerar su entrelazamiento irreducible con la totalidad de las expresiones en el lenguaje utilizado.

${ }^{7}$ La concepción del progreso teórico de Nagel, centrado en la idea de reducción homogénea de las teorías a sus sucesoras, al mostrar corresponder a una falsedad histórica, ayudó a consolidar las críticas holistas de Feyerabend y Kuhn al progreso epistémico.
} 
son entidades lógico-lingüísticas a la manera del híbrido mencionado. La otra motivación mayor es la antigua presunción de que ciertos conceptos y niveles teóricos descansan en "necesidades metafísicas" -idea ampliamente aceptada en las ciencias naturales hasta principios del siglo XX, todavía vigente en algunos sectores (Cordero, 2011b).

Desde fines del siglo XIX, sin embargo, los científicos (especialmente los físicos teóricos) transgreden rutinariamente los imperativos de la filosofía meta-científica. Un desarrollo clave en esta dirección fue la Teoría Especial de la Relatividad, en la cual Einstein presenta las redes conceptuales como relaciones contingentes -que no metafísicas - basadas en inducciones revisables en principio. Desde entonces, el fraccionamiento de teorías y redes conceptuales en componentes autónomas ha ganado terreno en las ciencias y la filosofía de la ciencia. Hoy, la idea de que las teorías fundamentales forman sistemas monolíticos irrompibles carece de sustento consensuado, y somos muchos los que pensamos que el holismo semántico y el necesitarismo metafísico descansan en mitos e inexactitudes acerca del lenguaje y el conocimiento. En general, las teorías se conciben ahora más como entendemos los mapas cartográficos que como sistemas de enunciados entrelazados entre sí por mega-estructuras holistas. A decir de pensadores como Philp Kitcher (2001) y Ronald Giere (2006), la concepción cartográfica de las teorías hace fácil conceder valor cognoscitivo autónomo a fragmentos teóricos y concentrar la actitud realista en estructuras parciales de diferentes grados de abstracción. Eso es precisamente lo que tratan de lograr los realismos selectivos, según los cuales una parte "fundamental" de, por ejemplo, la exitosa teoría original de Fresnel (centrada en el éter luminífero) estaba radicalmente equivocada. No obstante, dicha teoría contiene muchas partes acertadas a diversos niveles de descripción - como el "Núcleo de Fresnel", mencionado anteriormente. Este último, abstraído del hipotético sustrato material de las ondas luminosas, provee una descripción esquemática empíricamente exitosa y libre de dudas específicas, la cual todas las teorías sucesoras han retenido en primera aproximación.

\subsection{Descripción totalmente fiel}

ste ideal asume que tomar posición realista sobre una teoría implica aceptar que el mundo es exactamente como ella dice. Comprende tres variantes: (3a) el literalismo, (3b) el exactismo, y (3c) el exactismo metodológico.

(2a) Como en el fundamentalismo bíblico, el literalismo presume que cualquier error en una narración o explicación científica es excesivo e inabilitante. El impacto potencial es dramático. Muchas de las afirmaciones centrales de la teoría del flogisto yerran, y lo mismo ocurre con la teoría de Fresnel $-\mathrm{y}$ para tal caso con todas las grandes teorías empíricamente exitosas del pasado. Por consiguiente, según el literalismo, todas están radicalmente equivocadas. Los usos anti-realistas de esta manera de ver las teorías son diversos. Si cualquier 
divergencia entre dos hipótesis hace que cuenten como radicalmente distintas, entonces la probabilidad de que algún científico seleccione la teoría exactamente correcta entre todas las posibles es miserablemente pequeña (argumento de los "bad lots"). De otro lado, la probabilidad inicial de conjeturar "la" (única) teoría propiamente verídica es también irremediablemente ínfima (problema de la tasa básica). Y, por lo tanto, en cualquier momento dado, la probabilidad de que la única teoría verídica se encuentre entre las "alternativas ya concebidas" es desconsoladoramente baja.

Este tipo de literalismo pertenece a los cuentos de hadas. En la ciencia, el rompimiento con el literalismo ocurre bastante temprano, en el uso de idealizaciones explicativas -específicamente en la formulación de hipótesis sin expectativa de realización exacta en la naturaleza. Por ejemplo, la teoría cinética clásica de la materia trata de explicar causalmente las leyes empíricas descubiertas en siglos anteriores acerca del comportamiento macroscópico de los gases (e.g," $\mathrm{PV}=\mathrm{nRT}$ ) y diversos materiales (e.g., la expansión térmica). En el caso de los gases, la explicación apela a partículas puntuales carentes de estructura ("gases ideales"), que los teóricos involucrados no creían existiesen como tales. Boyle, en particular, sostenía que los gases reales consisten en corpúsculos voluminosos que se mueven al azar y se encuentran a distancias-promedio relativamente grandes entre sí. Las partículas puntuales invocadas en la teoría cinética son, pues, una idealización. Según el realismo, el comportamiento microscópico de los corpúsculos de que están hechos los gases instancian en grado significativo, pero limitado, el comportamiento de un gas ideal. Lo hacen al menos en algunas situaciones físicas discernibles, dentro de ciertos márgenes de error.

(2b) El exactismo asume que en la naturaleza hay propiedades cuantitativas de las cuales los sistemas concretos tienen valores definidos y que las afirmaciones derivables de una teoría correcta corresponden al mundo con exactitud total. Este "realismo de precisión" supone que las cantidades propuestas por los teóricos deben referir objetivamente del mayor modo imaginable. El exactismo armoniza con ideales intelectuales muy antiguos, pero que han perdido credibilidad.

En particular, la posición malinterpreta la forma como funciona la representación teórica (Paul Teller, 2015). Repitiendo lo dicho, las teorías generalmente formulan idealizaciones que presuponen el fracaso de sus atribuciones cuantitativas exactas en situaciones concretas. Por ejemplo, de modo literal estricto, la afirmación "la barra archivada en París como patrón del metro longitudinal tiene un metro de longitud" solo puede ser verdadera por definición: todo intento de comprobar la afirmación contra cualquier otra longitud objetiva externa se verá limitado por fluctuaciones térmicas y cuánticas ineliminables. El exactismo cuantitativo es un mito que no toma en cuenta el estado epistémico de la ciencia actual.

(2c) Por su parte, el exactismo metodológico sostiene que las teorías deben tratar de producir descripciones "exactas" de cómo son las cosas, so pena de va- 
ler únicamente como instrumentos. Esta manera de entender el objetivo de las teorías imagina a los científicos teóricos como una suerte de informantes comprometidos con aprehender la realidad de una manera totalmente libre de dispersiones, como la que tendría un dios omnisapiente en un mundo como el de la física clásica.

Este suplemento metodológico se basa en un mito que perdió credibilidad en el siglo XVIII. Todavía, sin embargo, algunas variantes contemporáneas (que repudian la ontología clásica pero no el ideal de visión divina), generan expresiones extáticas por parte de científicos a la caza de teorías que, según ellos sugieren, reflejarían la mente de Dios. Stephen Hawking es explícito al respecto: en su opinión, de formularse, la "Teoría del Todo" lograría realizar "el triunfo final de la

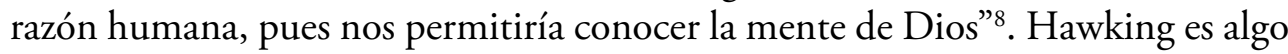
socarrón y pudiera estar bromeando, pero la idea de leer la mente de Dios todavía recurre mucho entre grandes teóricos. Desde la perspectiva del realismo selectivo, la tarea cognoscitiva va en otra dirección: ser realista sobre una teoría no implica creer que ésta dice "la verdad, toda la verdad, y nada que la verdad" acerca de algo, mucho menos que refleja "la mente de Dios". La actitud realista cobra sentido cuando partes selectas de una teoría ofrece logros cognitivos significativos, aunque éstos sean finitos, forjados con los medios imperfectos a disposición de los científicos. (Más sobre este particular en la sub-sección 5).

\subsection{Teorías mayormente correctas}

Según muchos pensadores, el realismo científico sostiene que "la mayoría de las entidades esenciales no-observables de las teorías científicas contemporáneas bien establecidas existen independientemente de la mente y tienen las propiedades que la ciencia les atribuye" (Devitt, 2005, 769).

Esta idea tienta mucho. Sin embargo, ignora hechos importantes -en particular, que normalmente las teorías exitosas se equivocan considerablemente, sobre todo a los niveles descriptivos más profundos? ${ }^{9}$ Por ejemplo, la Teoría del Alma Motriz del Sol, propuesta por Kepler hacia 1610 para explicar la persistencia de los movimientos de los planetas, hace predicciones correctas, como que el Sol gira en la misma dirección que las órbitas planetarias y lo hace más rápidamente que los planetas. Análogamente, en las teorías del calórico y el flogisto las entidades centrales postuladas resultaron ficticias. La exitosísima la teoría de la luz de Fresnel y el electro-magnetismo clásico demandaba la existencia de un medio, el éter, que luego los físicos desalojaron de sus teorías a principios del siglo pasado. Casos similares abundan no solo en la física. En embriología, no obstante, los importantes éxitos predictivos logrados por las teorías vitalistas del

${ }^{8}$ New Scientist, 4 de marzo de 2010.

${ }^{9}$ Ver, e.g., Lyons 2006, Stanford 2006, Cordero 2011, y Vickers 2013. 
siglo XIX, resulta que las supuestas bio-fuerzas no existen. La teoría de Kekulé de la molécula del benceno postulaba la existencia de dos formas de la molécula en equilibrio dinámico; la teoría mostró significativos éxitos, pero su error quedó claro cuando fue posible desarrollar modelos cuánticos en la química. Por su parte, la Teoría Cuántica de Bohr de 1912 predice los espectros del hidrógeno y el helio ionizado; lo hace a partir de órbitas electrónicas que, a la luz de resultados posteriores, parecen no existir. Las redes nomológicas y, en general, las estructuras modales de las teorías exhiben restricciones análogas, esbozadas en los acápites (4) y (5) a continuación. Hay conciencia de estas limitaciones en la práctica científica. Como apremiaba David Bohm a mediados del siglo XX, las cualidades, propiedades y categorías de regularidades de la materia expresadas mediante un conjunto finito de cualidades y leyes son generalmente aplicables solo dentro de contextos limitados, en términos de rangos de condiciones y grados ${ }^{10}$.

\subsection{Términos y supuestos “centrales"}

Otra idea frecuente sostiene que tomar una posición realista acerca de una teoría dada exige comprometerse con sus supuestos centrales (principios, entidades y leyes que la individualizan). La lectura escéptica de la historia de la ciencia de Laudan, en particular, asume que una teoría cuyos términos centrales carecen de referencia (como en los casos del flogisto y el éter) no puede tomarse realistamente.

Este supuesto compromiso con los términos centrales parte de ciertos mitos sobre las teorías, y también de algunas falsedades de moda. Para empezar, una teoría no versa exclusivamente sobre las entidades y relaciones "centrales" que figuran en ella al nivel más fundamental. Versa, también (argüiblemente en primer lugar), sobre el dominio que tiene por objeto, cuyas entidades y estructuras relevantes incluyen las postuladas por la teoría a niveles intermedios y superficiales de descripción, como el Núcleo de Fresnel ya mencionado.

Para el realista selectivo, los principios y entidades centrales de una teoría dada la individualizan, pero no agotan su valía epistémica. Adoptar una posición realista requiere identificar descripciones teóricas con expectativas de veracidad, pero éstas pueden ser de nivel intermedio. Correspondientemente, la atención realista se concentra en afirmaciones teóricas para las cuales hay evidencia fuerte, sin importar si los términos involucrados son o no "centrales" o "intermedios". Los éxitos de la óptica de Fresnel no bastaron para tomar realistamente la ontología inicial de la teoría (centrada en el éter luminífero), pero sí ciertas partes teóricas relativamente abstractas - como el Núcleo de Fresnel, debidamente restringido por cláusulas ceteris paribus (más sobre este particular en el siguiente acápite).

${ }^{10}$ Bohm (1957), Capítulo V. 


\subsection{Universalidad}

La noción de que las teorías científicas fundamentales deben ser universales ha tenido defensores prominentes en todas las épocas, incluyendo la actual. Para van Fraassen $(1980,86)$, por ejemplo, desde la perspectiva realista "una teoría no puede ser verdadera a menos que pueda extenderse consistentemente, sin corrección, a toda la naturaleza”. Por su parte, John Earman afirma que la universalidad nomológica va de la mano con la idea de unidad de la ciencia; las cláusulas ceteris paribus ineliminables - sostiene- amenazan la unidad de la ciencia y la primacía de la física fundamental sobre las ciencias especiales. En palabras de Earman, Roberts \& Smith (2002): “[A]demás de no haber de ejemplos legítimos en la física, sugerimos que enunciados que de suyo no serían problemáticos se vuelven inestables por la mera adición del operador Ceteris Paribus. Leído como una sencilla afirmación fáctica, ' $C P$ todos los $F$ s son $G s$ ' no puede ser materia de teorización científica”.

Hay argüiblemente mucho de mito y no poco de falso en estas exaltaciones de la universalidad nomológica. Como con la teoría de Fresnel del siglo XIX, por ejemplo, las leyes han mostrado corresponder a regularidades veraces solo dentro de ciertas condiciones físicas; su estatus hoy es el de leyes ceteris paribus: e.g., si la luz incidente es suficientemente intensa, las leyes de Fresnel muestran discrepancias dramáticas con la situación física; el efecto fotoeléctrico aparta dichas leyes aún más de la fundamentalidad al añadir restricciones teórico-cuánticas a la interacción entre la luz y la materia. Así pues, la exigencia de tomar las teorías como afirmaciones de alcance universal, sin restricciones, parece defendible solo a nivel metodológico. A los niveles ontológico y epistemológico, las tesis respectivas descansan en alegorías que, tomadas con fidelidad total, frustran la representación científica en general, no solo la posición realista.

El universalismo acerca de las leyes fundamentales continúa siendo materia de debate, pero sus críticos incluyen protagonistas de peso. Bohm (1957), por ejemplo, argumentaba que los conjuntos finitos de leyes simples pueden proporcionar descripciones y predicciones correctas cuando limitamos su contexto lo suficiente, pero - añadía - es de esperar que las teorías sin restricciones probablemente muestren ser falsas. Las leyes de Fresnel, las ecuaciones de Maxwell, y las de prácticamente todas las teorías del pasado, son falsas como generalizaciones sin excepciones. Pueden interpretarse como correctas solo en ciertas circunstancias físicas (que es cómo las utilizan los físicos). Al igual que las leyes de Fresnel, las ecuaciones de Maxwell han perdido conexión con su ontología original de referencia y se han incorporado variadamente a teorías posteriores como la Relatividad General y la Teoría de Campos Cuánticos.

La visión de Bohm es afín a otras en el campo. Según Heinz Post (1971), por ejemplo, las partes teóricas que sobreviven cambios teóricos radicales normalmente lo hacen reduciendo su alcance de aplicación y perdiendo el carácter de 
regularidades fundamentales. En tiempos recientes, Nancy Carwright defiende una posición consonante con estas concepciones. En The Dappled World dice lapidariamente:

[...] como lo sugieren las apariencias, vivimos en un mundo moteado, un mundo rico en cosas diferentes, dotadas de diferentes naturalezas, que se comportan de diferentes maneras. Las leyes que describen este mundo forman un mosaico, no una pirámide. No siguen la estructura simple, elegante y abstracta de un sistema de axiomas y teoremas. Más bien parecen $-\mathrm{y}$ se aferran firmemente a parecerse- a la ciencia tal como la conocemos: dividida en disciplinas, al parecer desarrolladas arbitrariamente; de modo que cada una gobierna diferentes conjuntos de propiedades a niveles de abstracción, con regiones de gran precisión, grandes parcelas de aforismos cualitativos resistentes a la formulación precisa; superposiciones erráticas; aquí y allá, de vez en cuando, esquinas que se alinean, pero en su mayoría lo que hay son bordes irregulares; y siempre la cobertura de la ley apenas ligeramente unida al mundo desordenado de las cosas materiales. $(1999,1)$

No parece, pues, haber razón convincente para negar legitimidad a las teorías que incluyen cláusulas ceteris paribus. Ninguna propuesta mayor de la ciencia estaría realmente libre de ellas. En cualquier caso, el proyecto realista va por otro lado. Para éste, la cuestión crucial es si las inferencias científicas conducen o no a aserciones muy probablemente veraces sobre aspectos del mundo inaccesibles a la percepción ordinaria - no importa lo unitario, bonito o feo, central o intermedio que sea el mundo así develado. Si esta apreciación es correcta, entonces resulta inadmisible elevar los estándares epistemológicos contra las teorías científicas que no calzan bien con el unificacionismo, el reduccionismo o el esteticismo. La actual imagen científica del mundo está hecha de retazos (al menos por ahora). Para el realista selectivo, el centro de atención son los contenidos con alta probabilidad de veracidad. Al respecto, las partes epistémicamente más confiables de la ciencia - en particular, las que han sobrevivido el triunfo de múltiples sistemas teóricos a lo largo del tiempo- rarísima vez son a todo nivel descriptivo exactamente las mismas que los científicos propusieron originalmente. En general, son versiones actualizadas, comparativamente abstractas, restringidas, de grano más grueso, y con múltiples realizaciones ontológicas a los niveles más profundos.

\subsection{Progreso teórico}

La posición realista suele asociarse con ideas optimistas acerca del progreso teórico, en particular expectativas de (a) progresismo lineal monotónico y (b) convergentismo panteórico. 
(6a) Según el progresismo lineal, los contenidos teóricos progresan sin retroceder, incorporando completamente los logros anteriores.

Este tipo de progresismo calza mal con la admisión, ampliamente compartida, de que, si las teorías exitosas aumentan el conocimiento, lo hacen equivocándose a diversos niveles. Esto parece claro en casos como el del Núcleo de Fresnel. Como veíamos en (2.5), desde su primera formulación en el siglo XIX, las leyes de Fresnel han venido incorporando cláusulas de restricción que las transfieren a niveles descriptivos cada vez más más abstractos, y/o les restringen el dominio de aplicación, y/o limitan el poder de resolución veraz. En el siglo XX, las modificaciones aludidas se suplementaron con elucidaciones y especificaciones a partir de teorías inicialmente externas (en particular relaciones inter-teóricas con la física cuántica). A pesar de lo cual, en primera aproximación, la descripción de la luz ofrecida por el aludido Núcleo de Fresnel ha permanecido constante a niveles nada triviales.

(6b) Convergentismo Panteórico. Según esta idea popular, la posición realista está metafísicamente comprometida con la existencia de una descripción del mundo verdadera y completa, cuyas aseveraciones guardan una relación biunívoca con la realidad independiente de la mente, y que el propósito de la ciencia es descubrir esa descripción.

Ningún realista serio ha mantenido semejante posición en generaciones. Se trata de una tesis sacada de la tumba en el último cuarto del siglo pasado por pensadores como Richard Rorty y Hilary Putnam. Putnam (1978; 1990), en particular, la ofrece bajo el nombre de "realismo metafísico", pero lo hace presentando dicha posición como ejemplo de proyecto sin esperanzas de realización (1978, 49; 1990, Prefacio).

\subsection{Realismo e inteligibilidad}

Los realistas tratan de justificar la extensión de inferencias ampliativas de la ciencia a contenidos teóricos, y en tal medida apuestan al meliorismo intelectivo. Los anti-realistas, por el contrario, apelando a consideraciones generales de prudencia epistémica, proponen limitar la inferencia ampliativa a contenidos accesibles a la percepción ordinaria, restringiendo así el alcance de las explicaciones científicas que consideran razonablemente creíbles. Como sugería en (1.4), desde la perspectiva realista, para rechazar una idea no basta con apelar a dudas generales sobre ella. Es insuficiente invocar la mera posibilidad de que sea falsa; hay que identificar dudas especificas relativas al caso. Esta diferencia en torno a los alcances de la inferencia científica justificable se extiende a las posiciones correspondientes sobre los alcances de la inteligibilidad científica. Para el empirista constructivo, por ejemplo, la única explicación razonable del éxito empírico de la ciencia es que las teorías se aceptan únicamente mientras no violan el objetivo de adecuación empírica. Los realistas objetan que esta propuesta explica, a lo mucho, por 
qué las teorías actuales se mantienen vigentes, no por qué son exitosas, dejando el éxito empírico gratuitamente hundido en el misterio. Desde la perspectiva realista, las teorías empíricamente exitosas lo son porque el mundo es aproximadamente como ellas dicen. El realismo, por consiguiente, va de la mano con el ideal de expandir nuestro entendimiento del mundo. Pero este ideal puede llevar a desvarío. El meliorismo intelectivo tiene una versión radical y otra moderada.

(7a) El Ideal de Entendimiento Radical exige que las teorías nos dejen intelectualmente satisfechos a cabalidad (completamente libres de "por qués" relevantes). Tal exigencia es constitutiva de la antigua filosofía natural a la que apela Leibniz para acusar a Newton de introducir fuerzas "ocultas" en la ciencia. Fiel a la época, hasta el final de su vida Newton aceptó el cargo a regańadientas, buscando una explicación "inteligible" de la gravitación que apelara solo a interacciones por contacto directo. El creciente éxito mostrado por la concepción menos exigente de filosofía natural defendida por los newtonianos ayudó a devaluar el ideal tradicional de inteligibilidad. En el siglo XIX, los científicos de tendencia empirista indultaron a Newton de su condena. A principios del siglo siguiente, Einstein otorgó ciudadanía científico-filosófica a la opacidad intelectiva parcial, especialmente a nivel fundamental, como ejemplifican en la Teoría Especial de la Relatividad el postulado de la velocidad de la luz como invariante universal y la omisión de un sustrato material explicativo para las ondas electromagnéticas. Las teorías científicas modernas ni proporcionan ni ofrecen inteligibilidad radical.

Los físicos, en particular, dejan preguntas abiertas, y la justificación científica (y la inteligibilidad) se detienen mucho antes de conferir nivel apodíctico a las aseveraciones teóricas. Rutinariamente, sin embargo, se aceptan como justificados contenidos de nivel teórico bastante alto, los cuales amplían el entendimiento de los dominios empíricos estudiados. La inteligibilidad lograda es ostensiblemente parcial, demarcada por fronteras. Sin embargo, dichas fronteras son epistémicamente contingentes, no corresponden a preferencias preestablecidas "metacientíficamente" (como, por ejemplo, las de los empiristas tradicionales). Desde la perspectiva del realismo (y la práctica científica común), el factor clave para tomar como creíble una parte o narrativa teórica dada no es la "inteligibilidad radical" que confiere. El factor decisivo es que la parte en cuestión (a) sea exitosa y tenga impacto diferencial sobre el poder predictivo de la teoría de que se trate, y (b) esté libre de dudas específicas en contra.

(7b) Intelección Finita. La versión moderada de la expectativa de inteligibilidad acepta la inclusión de "hechos brutos" en el discurso científico. No acepta, en cambio, que la identificación de tales hechos se haga a partir de consideraciones meta-científicas (como, por ejemplo, si los contenidos involucrados son o no de carácter observable). Especialmente en los realismos de corte naturalista, los criterios de delimitación son provisionales y descansan en razones científicas específicas. 
Esta versión moderada expresa una opción prescriptiva compatible con la práctica científica y la vida ordinaria: las "buenas" teorías deben ofrecer algo más que poder predictivo; deben lograr también que los fenómenos sistematizados se entiendan causalmente mejor. Puesto así, una ventaja del realismo frente al empirismo constructivista es que el realismo esclarece por qué las teorías seleccionadas por las ciencias maduras son empíricamente exitosas (porque, a niveles relevantes, el mundo es aproximadamente como dichas teorías dicen que es). Las posiciones no-realistas, en cambio, se limitan a explicar por qué dichas teorías reciben aceptación (porque son empíricamente exitosas), adoptando posiciones arbitrariamente misterianistas acerca de su éxito empírico.

\subsection{La gran ilusión}

Según una tesis con no pocos partidarios, los únicos realismos dignos de consideración son los que cumplen con la mayoría de las aspiraciones listadas anteriormente. En contra de esta idea, he tratado de mostrar que ninguna de las expectativas consideradas vale realmente la pena. Si las sugerencias anteriores al respecto se aceptan, entonces cada una de las aspiraciones consideradas carece de base convincente y sus versiones fuertes descansan todas en mitos, confusiones, y/o falsedades piadosas que tomadas literalmente resultan fatales.

Por añadidura, aun si las expectativas en cuestión convenciesen (cosa que no hacen), igual enfrentan esta dificultad mayor: desde la perspectiva del actual debate sobre realismo/antirealismo todas son irrelevantes, en la medida que ninguna marca contrastes epistémicos pertinentes entre contenidos de nivel pre-teórico y nivel teórico. Si el holismo es un problema para el discurso teórico, lo es también para el discurso observacional. Si cualquiera de los tipos de literalismo considerados crea problemas para atribuir verdad a las teorías, lo hace también en el caso de las representaciones observacionales. Si las teorías son verdaderas sólo en parte, lo mismo ocurre con las generalizaciones observacionales. Si muchos de los términos centrales de las teorías carecen de referencia, eso también ocurre con la referencia descriptiva de muchas generalizaciones observacionales. Si las regularidades teóricas están contaminadas por restricciones ceteris paribus, también lo están las regularidades observacionales. Y si los linajes teóricos no exhiben progreso epistémico lineal, tampoco lo hacen los linajes de discursos observacionales.

\subsection{Consideraciones finales}

En la disputa con los constructivistas empíricos y los defensores de la inducción de Laudan, los contenidos en discusión son las descripciones de presuntas realidades inaccesibles a la percepción ordinaria. Si las anteriores consideraciones son correctas, entonces resultan infundadas las sugerencias que acusan a los 
proyectos selectivistas de diluir la posición realista convirtiéndola en deleznable. Como con los mapas, el interés realista está en aquellas partes de las teorías que, con expectativa de veracidad libre de dudas específicas, describen dominios de interés.

Las desideratas discutidas en las secciones anteriores, que supuestamente deberían regir sobre las posiciones realistas y que los proyectos de realismo selectivo ignoran o debilitan, son perpendiculares al plano de las consideraciones propiamente realistas. Los argumentos pertinentes conciernen temas como, por ejemplo, los alcances y límites de la descripción científica de entidades y procesos fuera del alcance de la percepción humana natural, o la cuestión de si los métodos forjados a lo largo de la práctica científica permiten a las ciencias realizar dicho objetivo. El proyecto realista, particularmente en sus versiones selectivistas, promete avances al respecto. Dichos avances están supeditados a la superación de problemas técnicos como los mencionados al inicio de este artículo, pero no a la satisfacción de mitos o falsedades sobre lo que significa adoptar una posición realista. En particular, los selectivistas necesitan decirnos cómo identificar satisfactoriamente y delimitar sin arbitrariedad partes teóricas que ameritan interpretación realista - pero ese es otro problema.

\section{Referencias bibliográficas}

Bohm, David (1957). Causality and Chance in Modern Physics. London: Routledge \& Kegan Paul Ltd.

Bunge, Mario A. (2007). A la Caza de la Realidad. Barcelona: GEDISA.

Cartwright, Nancy (1999). The Dappled World. Cambridge: Cambridge University Press.

Cordero, Alberto (2001). "Realism and Underdetermination: Some Clues from the Practices-Up”, Philosophy of Science, 68S: 301-12.

Cordero, Alberto (2011a). "Darwin's Theory and Prediction”, en F. Minazzi (ed.), Evolutionism and Religion. Milano: Mimesis Edizioni: 79-94.

Cordero, Alberto (2011b). "Scientific Realism and the Divide et Impera Strategy: The Ether Saga Revisited”, Philosophy of Science, 78: 1120-1130.

Cordero, Alberto (2017). "Retention, Truth-Content and Selective Realism", en Evandro Agazzi (ed.), Scientific Realism: Objectivity and Truth in Science. Cham: Springer Nature (2017): 245-256.

Devitt, Michael (2005). "Scientific Realism", en The Oxford Handbook of Contemporary Philosophy, Frank Jackson and Michael Smith (eds). Oxford: Oxford University Press: 767-91. 
Devitt, Michael (1991). Realism and Truth, $2^{\text {nd }}$ ed. Princeton: Princeton University Press.

Feyerabend, Paul (1975). Against Method. London: New Left Books.

Giere, Ronald N. (2006). Scientific Perspectivism. Chicago: University of Chicago Press.

Hesse, Mary B. (1974). The Structure of Scientific Inference. Berkeley and Los Angeles: University of California Press.

Kitcher, Philip (1993). The Advancement of Science. Oxford: Oxford University Press.

Kitcher, Philip (2001). Science, Truth, and Democracy. Oxford: Oxford University Press.

Kuhn, Thomas S. (1962). The Structure of Scientific Revolutions. Chicago: University of Chicago Press. (1970, 2nd edition, with postscript).

Kuhn, Thomas S. (1977). The Essential Tension: Selected Studies in Scientific Tradition and Change. Chicago: University of Chicago Press.

Laudan, Larry (1981). "Confutation of Convergent Realism", Philosophy of Science (48): 19-49.

Laudan, Larry (1984). "Realism without the Real", Philosophy of Science (51): 156-62.

Leplin, Jarrett (1984). "Truth and Scientific Progress", en J. Leplin (ed.), Scientific Realism. Berkeley: University of California Press: 193-217.

Leplin, Jarrett (1997). A Novel Defense of Scientific Realism. Oxford University Press.

Lyotard, Jean-François (1979). La condition postmoderne: rapport sur le savoir. Paris: Minuit.

Lyons, Tymothy D. (2006). "Scientific Realism and the Stratagema de Divide et Impera". British Journal for the Philosophy of Science (57): 537-60.

McMullin, Ernan (2013). The Inference that Makes Science, en Zygon (48): 143191.

Post, Heinz R. (1971). "Correspondence, Invariance and Heuristics", en (S. French y H. Kamminga (eds.), Correspondence, Invariance and Heuristics: Essays in Honour of Heinz Post. Dordrecht: Springer-Science (Boston Studies in the Philosophy of Science, Vol. 148): 1-43.

Putnam, Hilary (1978). Meaning and the Moral Sciences. London: Routledge and Kegan Paul. 
Putnam, Hilary (1990). Realism with a Human Face (edited by James Conant). Cambridge, MA: Harvard University Press.

Psillos, Stathis (1999). Scientific Realism. London: Routledge.

Rosenberg, Alexander (2000). Darwinism in Philosophy, Social Science and Policy. Cambridge: Cambridge University Press.

Ruse, Michael (1979). The Darwinian Revolution. Chicago: The University of Chicago Press.

Saatsi, Juha (2005). "Reconsidering the Fresnel-Maxwell Case Study". Studies in History and Philosophy of Science (36): 509-38.

Saatsi, Juha and Peter Vickers (2011). "Miraculous Success? Inconsistency and Untruth in Kirchhoff's Diffraction Theory". British Journal for the Philosophy of Science (62): 29-46.

Shapere, Dudley (1984). "Objectivity, rationality, and Scientific Change". Proceedings of the Biennial Meeting of the Philosophy of Science Association: 637663.

Sokal, Alan and Jean Bricmont (1998). Fashionable Nonsense: Postmodern Intellectual' Abuse of Science. New York: Picador.

Stanford, P. Kyle (2006): Exceeding Our Grasp: Science, History, and the Problem of Unconceived Alternatives. Oxford: Oxford University Press.

Teller, Paul (2015). "Language and the Complexity of the World”. En prensa.

Van Fraassen, Bas (1980). The Scientific Image. Oxford: Oxford University Press.

Vickers, Peter (2013). “A Confrontation of Convergent Realism”, Philosophy of Science 80:189-211.

Votsis, Ioannis (2011). "Saving the Intuitions: Polylithic Reference", Synthese 180 (2): 121-37.

Whewell, William (1847). The Philosophy of the Inductive Sciences, Volume II, Section III — "Tests of Hypothesis". London: John W. Parker.

Worrall, J. (1989). "Structural Realism: The Best of Both Worlds?”, Dialectica (43): 99-124. 
